\title{
ACE in neutrophil antibacterial defence
}

Angiotensin-converting enzyme (ACE) inhibitors are widely used to lower blood pressure and slow the progression of renal disease. New research, however, has revealed an important role for ACE in the antibacterial effectiveness of neutrophils. "ACE can do many things that affect the immune response," says Kenneth Bernstein. "We have previously shown that mice that overexpress ACE in monocytes and macrophages have an increased antitumour response and enhanced resistance to infection."

To assess the role of neutrophil ACE, Bernstein and colleagues studied the effect of ACE deletion and overexpression on neutrophil function in response to infection. ACE-knockout mice and mice treated with an ACE inhibitor were more susceptible to infection with methicillin-resistant Staphylococcus aureus (MRSA) than were wild-type mice, whereas mice with neutrophil-specific overexpression of ACE ( $\mathrm{Neu}^{\mathrm{ACE}}$ ) had increased resistance to MRSA and demonstrated better in vitro killing of MRSA and other bacterial species. Further examination showed that $\mathrm{Neu}^{\mathrm{ACE}}$ neutrophils had increased production of reactive oxygen species (ROS) following MRSA challenge and that inhibition of ROS attenuated the enhanced antibacterial resistance of $\mathrm{Neu}^{\mathrm{ACE}}$ neutrophils to MRSA. Neu ${ }^{\text {ACE }}$ neutrophils also demonstrated greater neutrophil extracellular trap formation and IL- $1 \beta$ release in response to MRSA than wild-type neutrophils.

"We think the key to the greater antibacterial activity of $\mathrm{Neu}^{\mathrm{ACE}}$ neutrophils is the production of superoxide by NADPH oxidase when challenged with bacteria," explains Bernstein. "This effect is due to ACE catalytic activity but does not seem to be due to angiotensin II. The implication is that $\mathrm{ACE}$ overexpression turns on some unknown pathway that strongly enhances myelomonocytic cell function. Find the unknown ACE substrate or pathway that is being activated and you have a totally new way to strongly increase the immune response of neutrophils and macrophages."

Susan J. Allison

ORIGINAL ARTICLE Khan, Z. et al. Angiotensin converting enzyme enhances the oxidative response and bactericidal activity of neutrophils. Blood http://doi.org/10.1182/blood2016-11-752006 (2017) 